\title{
A Primary Care-Based Quality Improvement Initiative to Increase Identification of Pediatric International Travelers
}

\author{
Jeffrey I. Campbell, ${ }^{1 \star}$ Andrea Alonso, ${ }^{2} \dagger$ Karen Cuttin, ${ }^{2} \dagger$ Miralia Sanchez, ${ }^{1}$ Sandra Schumacher, ${ }^{1}$ Al Ozonoff, ${ }^{1,3}$ \\ Alexandra Epee-Bounya, ${ }^{1}$ Tanvi Sharma, ${ }^{1}$ and Lara Antkowiak ${ }^{1}$ \\ ${ }^{1}$ Boston Children's Hospital, Boston, Massachusetts; ${ }^{2}$ Boston University School of Medicine, Boston, Massachusetts; ${ }^{3}$ Department of Pediatrics, \\ Harvard Medical School, Boston, Massachusetts
}

\begin{abstract}
Children who travel internationally to visit friends and relatives (VFRs) are at risk for travel-related illness, but underuse pretravel health services. Although primary care clinics can identify travelers and address pretravel health needs, to date, there are few published reports on effective primary care-based pretravel interventions. We developed a quality improvement initiative to increase traveler identification at a primary care clinic serving families that frequently travel to VFRs. Interventions included a screening question asked at all clinic visits, provider and staff training, travel fliers, and health recommendation sheets for families. Interventions were implemented during 2017 and 2018 peak travel seasons. Travel visit rates and characteristics during the intervention period were compared with pre-intervention baseline periods (April-August, 2015-16). Surveys with providers were conducted to assess disruptiveness of the interventions, and rates of duplicate travel visits were assessed. A total of 738 unique travel events were identified during peak travel seasons from 2015 to 2018 , encompassing travel to 29 countries across five continents. Overall, there were 428 unique travel events (3.0\% of all clinic visits) during peak seasons $2017-18$, compared with 310 unique travel events (2.2\% of all clinic visits) during peak seasons 2015-16 (rate ratio 1.34 [95\% Cl: 1.16-1.56], $P<0.001$ ). None of the 18 healthcare providers or staff surveyed found new travel screening processes to be disruptive or bothersome. Implementation of a primary care-based multimodal travel screening and education initiative was associated with a significantly increased rate of travel visits.
\end{abstract}

\section{INTRODUCTION}

Foreign travel, particularly to low-resource settings, exposes travelers to a number of health risks including infectious diseases, automobile accidents, and political violence. Between $22 \%$ and $64 \%$ of travelers report an illness while traveling, depending on destination. ${ }^{1}$ Although most of these are self-limited, ${ }^{2}$ severe illnesses can occur. ${ }^{3}$ Fortunately, many travel-related illnesses are preventable through immunization, chemoprophylaxis, and effective counseling. Pretravel consultation with health providers decreases morbidity in travelers. ${ }^{4}$ One group of travelers-those traveling to "visit friends and relatives" ("VFR," defined as any traveler whose purpose of travel is to visit friends or relatives)experience relatively high rates of travel-related illness. ${ }^{5}$ However, in a study of travelers returning with illness, VFR travelers were found to have comparatively low rates of pretravel healthcare consultation than non-VFR travelers. ${ }^{3}$ Children have been reported to represent a higher proportion of VFR travelers than adults. ${ }^{6,7}$ One study of patients attending Boston-area travel clinics found that $46 \%$ of VFR travelers visiting these clinics were $<5$ years old, compared with $<5 \%$ of non-VFR travelers. ${ }^{8}$

Potential barriers to pretravel consultation among VFR travelers include general lack of access to healthcare, lack of English language proficiency, likelihood of traveling on short notice, financial barriers, and health beliefs among this diverse group of patients and families. ${ }^{6,9,10}$ Several authors have proposed routine screening for travel in primary care settings that serve patients who are likely to travel to visit friends and relatives, such as clinics with large immigrant populations. $8,11,12$

Using primary care visits to identify and address travelrelated needs is an appealing strategy to identify pediatric VFR

*Address correspondence to Jeffrey I. Campbell, Division of Infectious Diseases, Boston Children's Hospital, 333 Longwood Ave., 6th Floor, Boston, MA 02115. E-mail: jeffrey.campbell@childrens. harvard.edu

†These authors are both co-second authors. travelers as well as others who may be undertaking relatively high-risk travel, given the frequent contact with primary care providers most children have in the first years of life and the importance of routine vaccinations in preventing travel-related illness. In this quality improvement initiative, we designed and implemented an intervention to increase screening for international travel in an urban pediatric primary care center serving a large immigrant population in the Boston area.

\section{METHODS}

Boston Children's Primary Care at Martha Eliot is a community-based primary care clinic in Jamaica Plain, Massachusetts, affiliated with a local major pediatric referral hospital. The center provides a broad range of services including primary care pediatric and adolescent clinics, a pediatric mental health clinic, and wraparound services such as nutrition and social work. There are typically around 13,000 pediatric visits each year; approximately $55 \%$ of families receiving care at the clinic list Spanish as their primary language. Given its catchment area, the clinic serves a large population of children who are either immigrants themselves or whose families recently immigrated to the United States.

In the fall of 2016, the clinic identified that improvement in travel-related services was a major need for its patients in the pediatric clinic. To further characterize need and variability in travel-related practice, we conducted both formal and informal interviews with providers (nurse practitioners, residents, and attending physicians), nurses, and support staff. From these interviews, several barriers to optimal pretravel care were identified, which guided subsequent intervention development: 1) lack of identification of travelers before clinic visits, 2) short time between clinic visits and departure date (limiting options for malaria prophylaxis and travel clinic referral), 3) lack of time during clinic visits to comprehensively address travel risks, and 4) patient/family lack of knowledge or concern about travel risks. Barriers to optimal travel care in our clinic, including those 
identified in interviews and from our own experience, are summarized in Figure 1. Of note, because of inconsistent documentation in patients' charts, we were unable to assess whether patients' primary goal of travel was to visit friends and relatives, although our experience working with patients at this clinic suggests that a substantial proportion of these patients were VFR travelers.

We sought to characterize recent historical travel visits to further identify areas of need. Specifically, we reviewed records of all clinic patients $<14$ years of age who presented to the clinic between April 2015 and August 2016 and who we identified as traveling. To identify patients who were traveling, we searched medical records for travel-specific interventions: prescription of malaria prophylaxis, administration of typhoid vaccination, or administration of measles/mumps/rubella vaccination in children $<1$ year of age. ${ }^{1,6}$ We also searched our electronic medical record system using a text visit indicator listing "travel" as a reason for the visit.

We then manually reviewed all identified records to determine the date of the travel visit, age of patient on the date of visit, and timing, duration, and location of travel, when documented. In addition, we analyzed the type of visit at which travel was discussed, whether or not the visit was "flagged" as travel related before patient arrival in the medical record's visit-reason text field, and the number of additional visits at which travel was documented as being addressed before travel. Because our unit of analysis was the travel visit, a single patient who traveled multiple times during the reference period could contribute data to multiple index visits. Although our clinic sees patients through age 26 years in its adolescent clinic, we only reviewed records for patients $<14$ years of age to ensure that patients in the pediatric clinic-where travel-related interventions were subsequently implemented-would be included in project monitoring and evaluation.

Based on our initial qualitative needs assessment, we aimed to increase the absolute number and rate (total travel-related visits/total pediatric clinic visits) of patients for whom upcoming international travel was identified in the primary care clinic. Our primary process measure was the number of visits for which travel consultation was the primary purpose ("dedicated travel visits"), compared with the number of visits for other purposes during which travel was also discussed. A second process measure was clinicians' perceived change in frequency of discussing travel with patients following introduction of the interventions. We included two balancing measures. First, we counted the number of repeated travel-related visits before a single trip, to gauge if screening patients before every visit resulted in duplicative travel consultation. Second, we assessed whether providers and staff found interventions to be disruptive.

Following baseline data collection and determination of key outcomes, we identified high-volume seasons lasting between April 1 and August 31, during which approximately $60 \%$ of annual travel visits occurred. This period roughly corresponded to Boston-area school summer holidays. This "peak travel season" provided natural periods to perform plan-do-study-act cycles: we planned interventions before each peak travel season, implemented them during peak season, and then studied outcomes and revised interventions during off-seasons.

The first intervention period took place between April 1 and August 31, 2017. To enhance identification of travelers, we implemented a single screening question: "Do you plan to travel outside of the country in the next 6 months?" Clinical assistants asked this question at the beginning of every patient visit to the pediatric clinic. To notify clinical providers when the travel screen was positive, as well as provide general travel guidance, we developed a single, half-page sheet with general travel recommendations, as well as specific recommendations based on the CDC's guidance for travel to the Dominican Republic, where most patients in our clinic population traveled. This informational sheet was printed on bright green stationary to be easily identifiable, and was included in the paperwork delivered

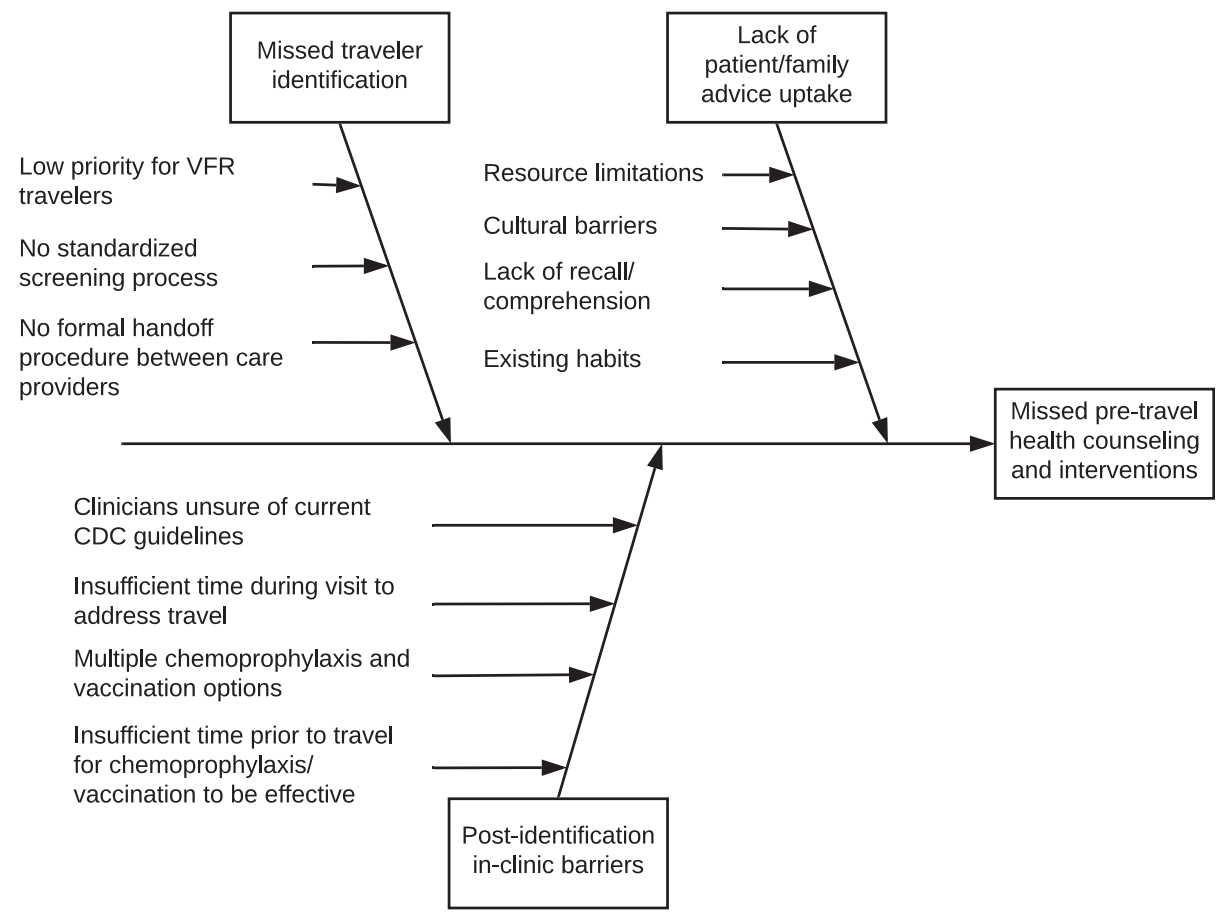

FiguRE 1. Driver diagram of barriers to effective pretravel care at our clinic. 
to providers after a patient was brought to their examination room. Finally, we hung posters in all pediatric examination rooms with pictures and instructions (in English and Spanish) requesting families to notify their providers of any future travel plans. During intervention cycle 1, we conducted informal interviews with clinic providers and staff about ways to optimize travel screenings and travel information sheets. We also traced each step of the process by which patients were screened and travel notification sheets were distributed to providers to identify methods to improve travel screening.

Following intervention cycle 1, we collated observations and suggestions about how to improve travel screening procedures and travel counseling. This led to several modifications of our intervention: 1) updated travel information sheets for providers that included specific information about malaria prophylaxis formulation and dosing, recommendations about prescriptions of antibiotics for travelers' diarrhea, and recommendations about post-travel tuberculosis testing; 2) development of new travel information sheets for patients in both English and Spanish for both general travel and travel to the Dominican Republic; and 3) lectures on travel medicine given at clinic staff meetings with specific guidance for clinic staff and resident physicians. Following these changes, intervention cycle 2 was implemented from April 1 to August 31, 2018.

Following each peak travel season, we identified and reviewed charts of all patients who had traveled since prior data review, using the same rules and metrics to identify and characterize travel visits during the baseline period. Thus, at the completion of peak travel season on August 31, 2018, we had reviewed and characterized all travel visits between April 1, 2015 and August 31, 2018 for patients < 14 years of age attending the health center. These data were divided into preintervention (April 1, 2015-August 31, 2016), cycle 1 (April 1August 31, 2017), and cycle 2 (April 1-August 31, 2018) periods.

During the fall of 2018, we conducted surveys with physicians/nurse practitioners $(n=9)$, nurses $(n=4)$, and clinician assistants $(n=5)$ working at the clinic to determine attitudes toward our interventions and providing travel care. The survey consisted of multiple choice, Likert-scaled, and free-response questions about acceptability and disruptiveness of the interventions, changes in travel practice, and perceptions of barriers and facilitators to travel care. Surveys were tailored to each professional category. Figure 2 summarizes the overall timeline of planning, implementation, and assessment of interventions.

We used frequency distributions and descriptive statistics to summarize quantitative travel and acceptability data. Pre-post comparisons were made using Fisher's exact test for proportions or Poisson regression for rates. Rates of travel visits for each month were calculated as total travel visits divided by total clinic visits for patients $<14$ years old. Rate analysis was restricted to peak travel seasons. Because we used retrospective chart reviews of free-text documentation, there were substantial missing or partial data in our dataset. Missing data were excluded from our analysis. Quantitative analysis was conducted using $R$ version 3.2. Free-response questions from staff/provider surveys were coded and analyzed for themes.

This quality improvement initiative was approved by the Boston Children's Hospital Department of Medicine Performance Excellence Group (the quality improvement council). Because this project constituted quality improvement rather than human subject research, the council adjudicated that the project did not fall under the authority of the institutional review board.

\section{RESULTS}

We identified a total of 986 initial (i.e., not duplicate) travel visits that occurred between April 1, 2015 and August 31, 2018 among patients $<14$ years old, which represented $1.6 \%$ of all visits to the pediatric clinic of the health center. Of all travel visits, the analytic sample consisted of 738 (75\%) visits, which included all travel visits occurring during peak travel seasons (April 1-August 31), summarized in Table 1. Among travel visits with documented durations of travel, the median duration was 3 weeks (range: 0-56 weeks; interquartile range: 24 weeks). Country-specific destinations were documented for 703 of these patients. During peak travel season, the leading destination of travel was the Dominican Republic, although several international destinations were represented. Four patients presented for visits to discuss travel to U.S. states, and 28 presented before travel to Puerto Rico.

During peak travel seasons in the retrospective baseline period (2015 and 2016), we identified 310 travel visits. In 2015

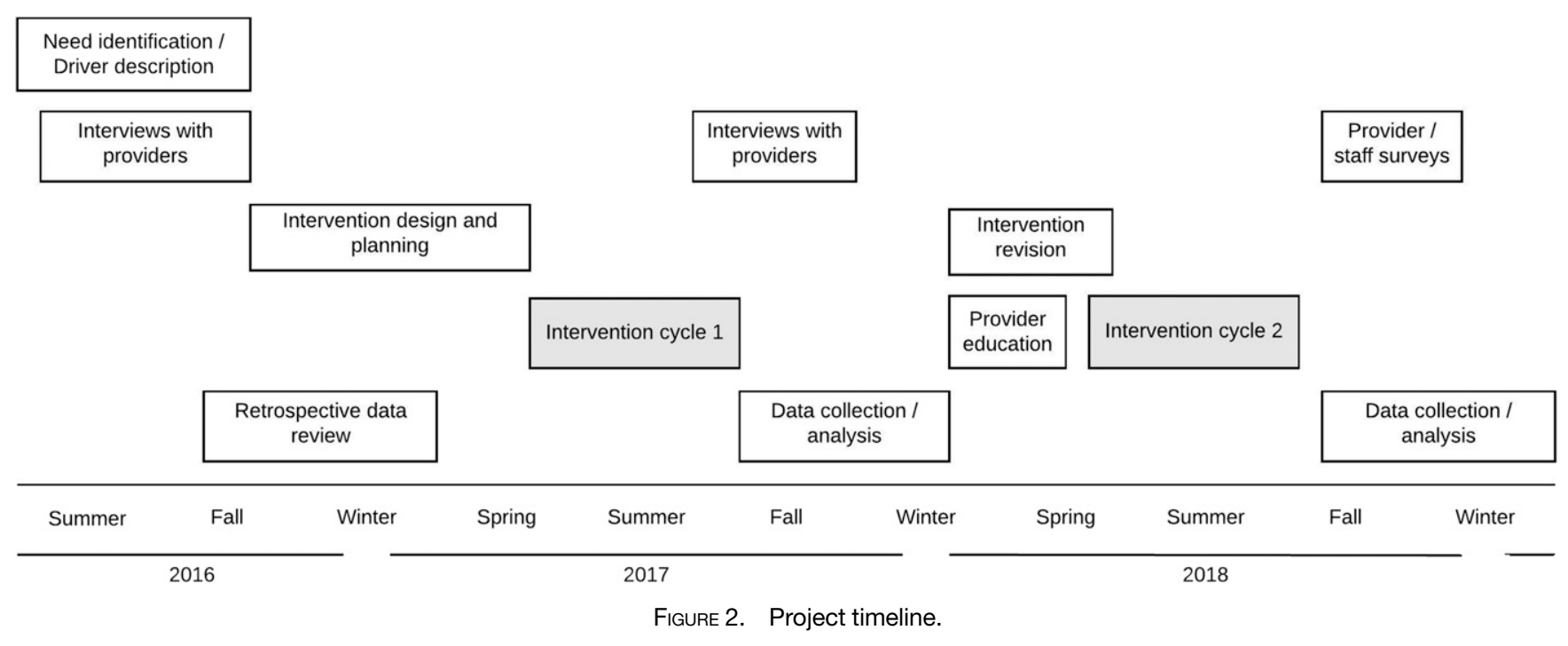


TABLE 1

Travel visit and traveler characteristics

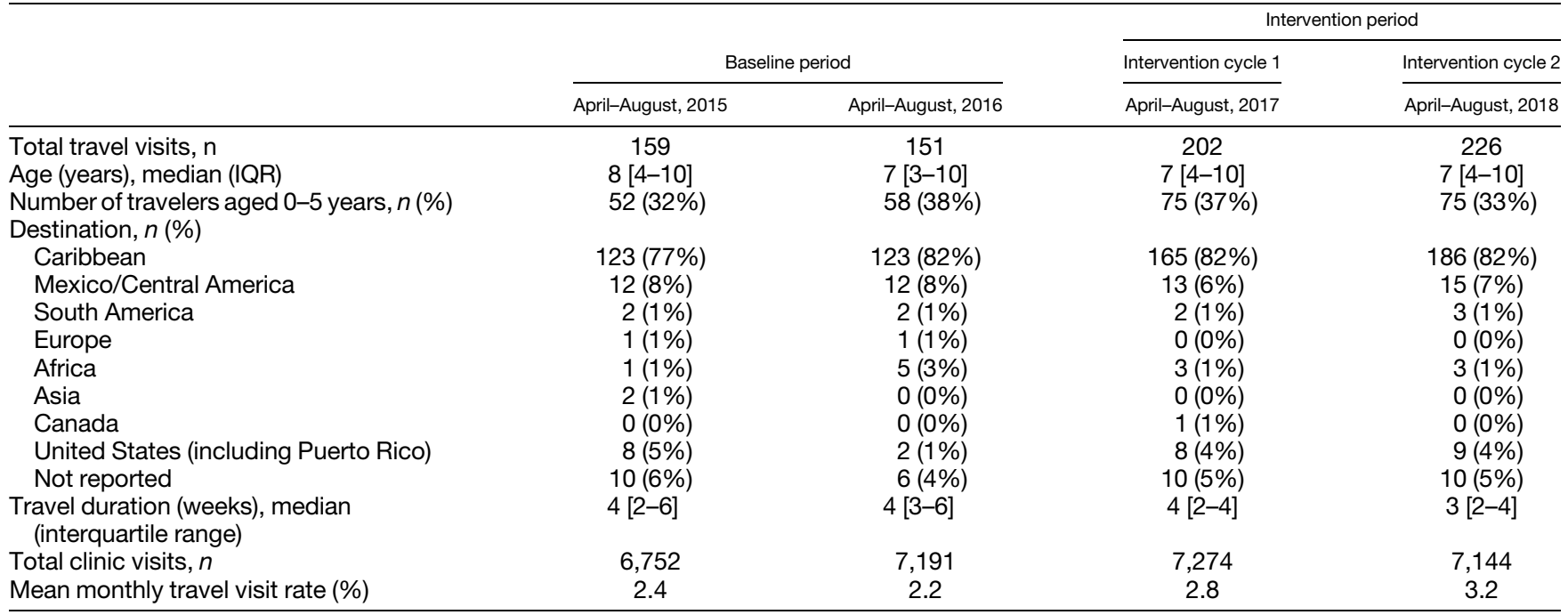

and 2016, the overall rates of travel visits per total clinic visits during peak travel seasons were $2.4 \%$ and $2.2 \%$, respectively. Following introduction of quality improvement measures in April 2017, we identified 428 travel visits that occurred in peak travel seasons of 2017 and 2018 (Figure 3). In 2017 and 2018, the overall rates of travel visits per total clinic visits during peak travel seasons increased to $2.8 \%$ and $3.2 \%$, respectively. Compared with the baseline period, the rate of traveler identification following quality improvement interventions increased by $34 \%$ compared with the pre-intervention period (rate ratio 1.34 [95\% Cl: 1.16-1.56], $P<0.001$ ).

The proportion of dedicated travel visits compared with all visits at which travel was addressed remained stable from the pre-intervention period to the intervention period (64\% versus
$62 \%$, respectively). Similarly, there was minimal change in other types of visits during which travel was addressed (Figure 4). In surveys with clinicians (physicians and nurse practitioners), six of eight respondents (75\%) reported screening for and discussing travel more frequently with patients following introduction of the travel interventions ( 1 clinician did not respond to this question).

There was minimal change in the number of repeated travelrelated visits between the baseline and intervention periods ( 8 versus 12 visits, respectively). All providers surveyed found the interventions to be useful in their practice, and none found the interventions to be disruptive to workflow. Follow-up surveys identified key barriers to effective pretravel care, including insufficient time to fully address travel during routine

Total and Percent Travel Visits

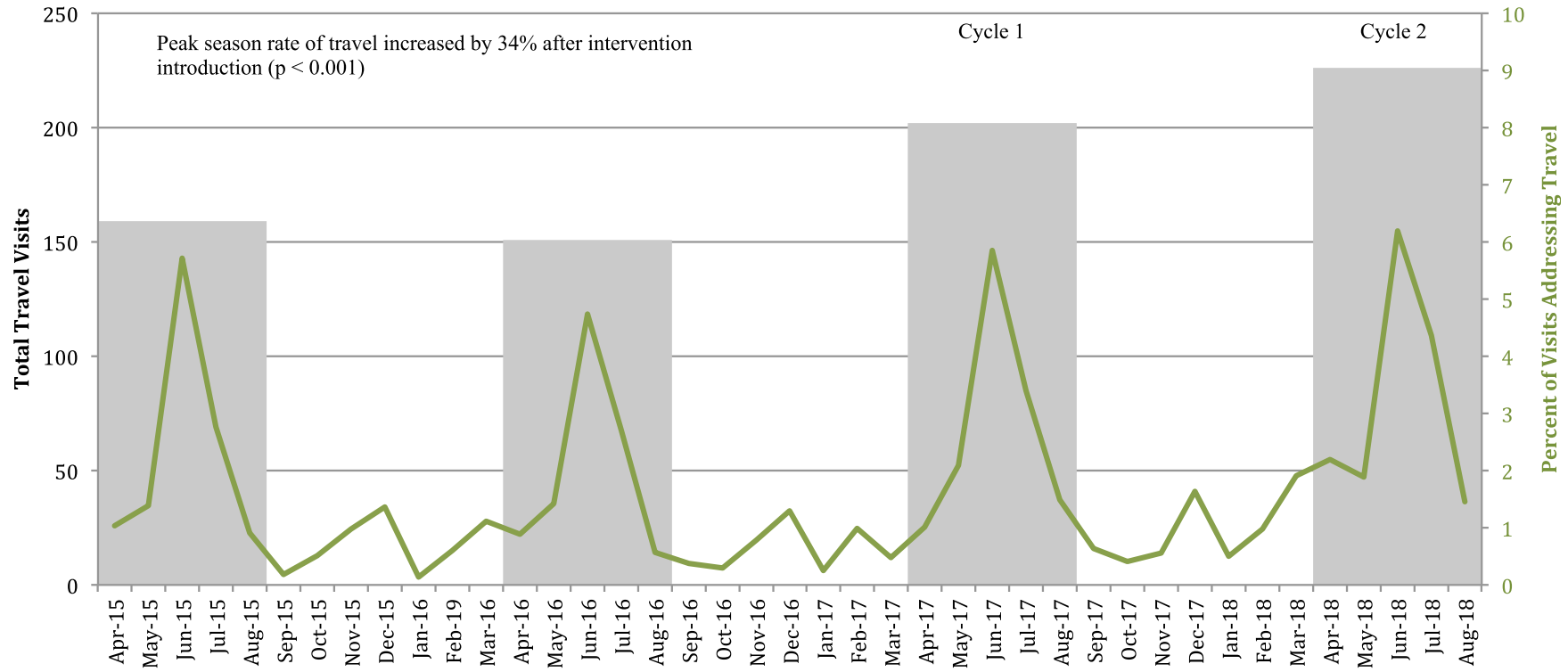

FIGURE 3. Total (gray bars, left axis) and percent of total visits in which travel was addressed (green line, right axis) over time. This figure appears in color at www.ajtmh.org. 
Types of Visits Addressing Travel

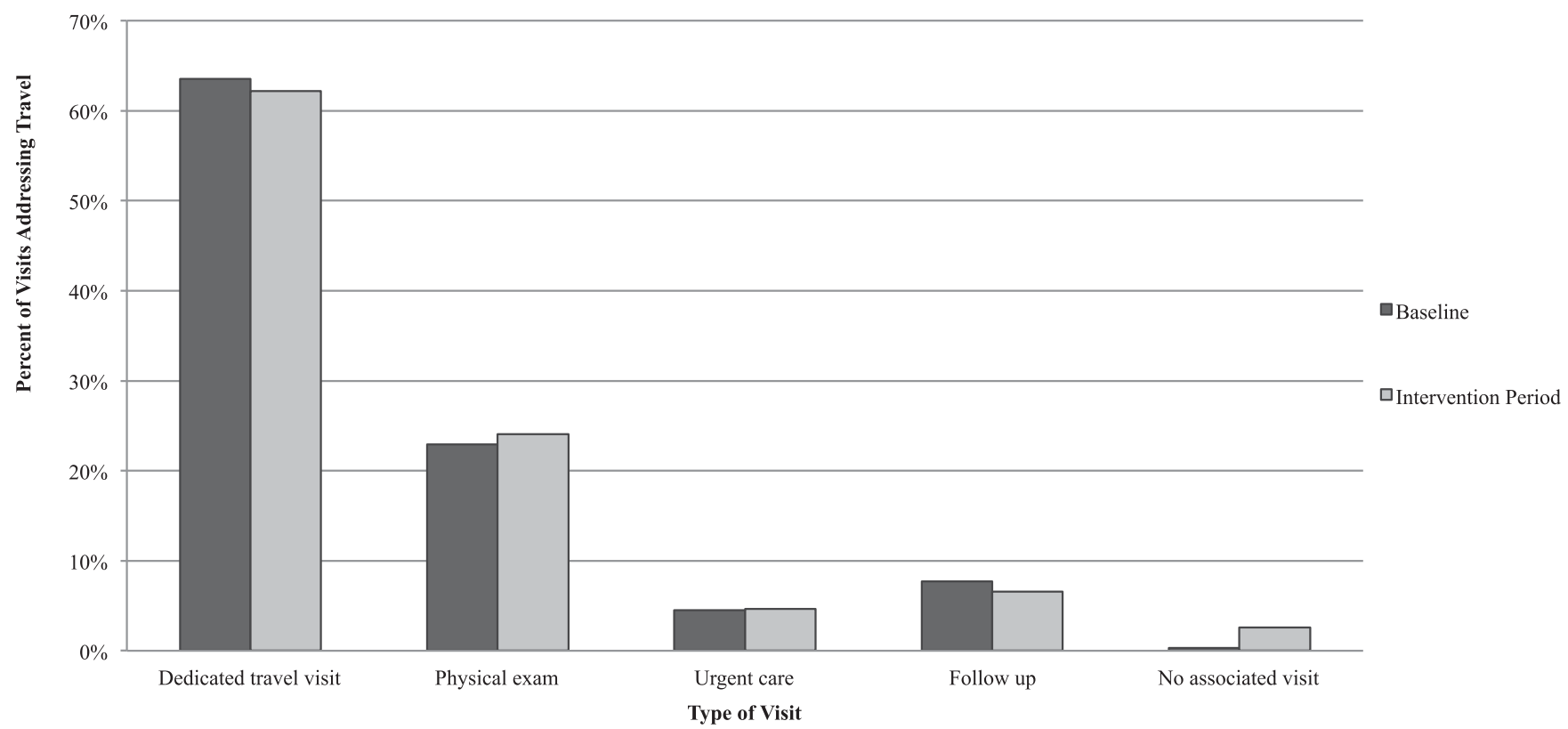

FIGURE 4. Visits during which travel was addressed.

appointments, poor patient understanding of travel health, and insufficient provider knowledge about risks of travel and how to address them (Table 2).

\section{DISCUSSION}

Based on identified and theorized barriers to effective pretravel care, we introduced tools to screen for upcoming travel and provide rapid pre-travel advice. Over the course of two intervention cycles, the rate of traveler identification significantly increased.

Although a number of innovative strategies have been evaluated to improve pretravel identification, a recent review highlighted the need for enhancing primary care-based travel care, particularly for VFR travelers. ${ }^{13}$ As results from prior studies have suggested, primary care centers serving large immigrant populations play an important role in identifying VFR travelers. ${ }^{8,12}$ Relevant to our Boston-area population, a survey of departing travelers from Boston's General Edward Lawrence Logan International Airport found that 38\% of travelers sought travel information from primary care providers, compared with $30 \%$ who sought travel information from travel medicine specialists. ${ }^{10}$ Among VFR travelers, 57\% sought information from primary care providers, compared with only $3 \%$ from travel medicine specialists. Although specialized travel clinics may be overall cost-saving for large health systems, ${ }^{14}$ we found that even with referrals to travel clinics, our patients often did not attend visits. This finding underscores the importance of strengthening primary care providers' capacity to address travel needs among high-risk patients.

Our patient population was well suited for primary care travel interventions. First, a majority of our patients traveled to the Caribbean (primarily to the Dominican Republic) or Central America, allowing us to create instruction sheets for providers that were tailored to these regions. Importantly, routine travel to these areas does not require specialized vaccination that could only be obtained at a specialized travel clinic (e.g., yellow fever or Japanese encephalitis). Second, after using the travel sheets, we anecdotally found that providers became more familiar with CDC recommendations for travel to common locations. For example, they became more aware of which areas of the Dominican Republic required malaria prophylaxis. Third, because our pediatric patients traveled primarily during school holidays, we were able to implement seasonal interventions targeted toward months with the highest frequency of travel visits. This may have reduced the alert fatigue that constant, year-round reminders could have generated.

TABLE 2

Barriers to effective pretravel care

\begin{tabular}{|c|c|}
\hline Barrier & Example quotes \\
\hline Insufficient time during appointments & $\begin{array}{l}\text { "Time-especially if a family is here for an urgent care appointment, need teaching } \\
\text { related to that visit and further teaching, vaccines, etc. for travel." (Physician) }\end{array}$ \\
\hline $\begin{array}{l}\text { Poor patient/family understanding of } \\
\text { travel health risks }\end{array}$ & $\begin{array}{l}\text { "Lack of knowledge by the family and some staff on the importance of planning the travel } \\
\text { visit ahead of time." (Nurse practitioner) }\end{array}$ \\
\hline $\begin{array}{l}\text { Insufficient knowledge about travel } \\
\text { medicine and risks }\end{array}$ & $\begin{array}{l}\text { "I feel like my knowledge about travel-related risks is limited and thus I'm not sure what } \\
\text { to include in my counseling." (Physician) }\end{array}$ \\
\hline Other barriers & $\begin{array}{l}\text { "Competing clinical concerns (socially/medically complex kids)." (Physician) } \\
\text { "Insurance coverage of specific medications." (Nurse practitioner) }\end{array}$ \\
\hline
\end{tabular}


Overall, we found that our quality improvement initiative was highly acceptable to physicians, nurses, and clinical assistants. Importantly, providers did not feel that the interventions disrupted their workflow, although some providers noted that they did not feel that they had enough time to address travel during routine clinic visits. As part of our traveler identification plan, we recommended that providers schedule dedicated travel visits when upcoming travel was identified during nontravel visits and could not be addressed at that time. However, we found that the types of visits at which travel was addressed did not change substantially between the pre-intervention and intervention periods. A number of factors could have contributed to minimal change in travel visit scheduling, including concerns about whether the family would return for a travel visit, insufficient time for a travel visit before departure, or the desire to address travel as soon as it was identified.

There are several limitations to our initiative. First, although the absolute number of travel visits and the rate of travel visits per total visits increased in our intervention cycles, we were by definition unable to measure the total pool of travelers because it is likely that many never came to the attention of clinicians. Second, the long duration of time between annual travel cycles limited the number of comparison units (each summer travel period) that we were able to analyze, thereby prohibiting us from accounting for unmeasured confounding or secular trends in travel behavior. Third, assessment of the disruptiveness of the interventions was based on a limited number of surveys with providers. We were unable to gather quantitative indicators of disruptiveness (e.g., time spent reviewing the travel guidelines that we provided). Fourth, because we used chart reviews to extract data, we were unable to confirm the proportion of travelers who were VFR, although given travel patterns and our own experience in our clinic, the proportion was likely quite high. Fifth, although we did assess the impact of our interventions on healthcare providers, we did not assess the impact on patients or families. Finally, because rates of travel-related illness are low in our population, we did not use travel-related morbidity or mortality as endpoints. Future interventions to enhance travel-related services for our population are being considered and may include interventions to improve tuberculosis testing, vaccination administration, and malaria prophylaxis prescription.

\section{CONCLUSION}

In this primary care-focused quality improvement initiative targeted at pediatric patients traveling to visit friends and relatives, our interventions were associated with an increased frequency and rate of traveler identification and increased adherence to recommended pretravel interventions. Strategies such as ours could be valuable to other pediatric primary care centers, particularly those serving a large population of VFR travelers. Our results also provide insight into travel behaviors of pediatric VFR travelers who often do not come to the attention of travel clinics or traditional travel research. Finally, our results highlight the role that community-based primary care clinics can play in providing timely, effective, and tailored pretravel guidance and interventions for high-risk pediatric travelers.

Received August 26, 2019. Accepted for publication December 22, 2019.
Published online March 2, 2020.

Acknowledgments: We would like to thank the patients and staff of Boston Children's Primary Care at Martha Eliot.

Financial support: This project was supported by a Boston Children's Hospital Patient Safety and Quality Graduate Medical Education grant.

Authors' addresses: Jeffrey I. Campbell, Miralia Sanchez, Sandra Schumacher, Alexandra Epee-Bounya, Tanvi Sharma, and Lara Antkowiak, Boston Children's Hospital, Boston, MA, E-mails: jeffrey.campbell@ childrens.harvard.edu, miralia.sanchez@childrens.harvard.edu, sandra. schumacher@childrens.harvard.edu, alexandra.epee-bounya@childrens. harvard.edu, tanvi.sharma@childrens.harvard.edu, and lara.antkowiak@ childrens.harvard.edu. Andrea Alonso and Karen Cuttin, Boston University School of Medicine, Boston, MA, E-mails: alonsoan@ bu.edu and kcuttin@bu.edu. Al Ozonoff, Boston Children's Hospital, and Department of Pediatrics, Harvard Medical School, Boston, MA, E-mail: al.ozonoff@childrens.harvard.edu.

\section{REFERENCES}

1. Freedman DO, Chen LH, Kozarsky PE, 2016. Medical considerations before international travel. $N$ Engl J Med 375: 247-260.

2. Jensenius $M$ et al., 2013. Acute and potentially life-threatening tropical diseases in western travelers-a GeoSentinel multicenter study, 1996-2011. Am J Trop Med Hyg 88: 397-404.

3. Leder K et al., 2013. GeoSentinel surveillance of illness in returned travelers, 2007-2011. Ann Intern Med 158: 456-468.

4. Schlagenhauf $P$ et al., 2015. Travel-associated infection presenting in Europe (2008-12): an analysis of EuroTravNet longitudinal, surveillance data, and evaluation of the effect of the pre-travel consultation. Lancet Infect Dis 15: 55-64.

5. Angell SY, Cetron MS, 2005. Health disparities among travelers visiting friends and relatives abroad. Ann Intern Med 142: 67-72.

6. Hendel-Paterson B, Swanson SJ, 2011. Pediatric travelers visiting friends and relatives (VFR) abroad: illnesses, barriers and pretravel recommendations. Travel Med Infect Dis 9: 192-203.

7. Maltezou HC, Pavli A, Spilioti A, Katerelos P, Theodoridou M, 2012. Paediatric international travellers from Greece: characteristics and pre-travel recommendations. Travel Med Infect Dis 10: 135-139.

8. Han $P$ et al., 2012. Health challenges of young travelers visiting friends and relatives compared with those traveling for other purposes. Pediatr Infect Dis J 31: 915-919.

9. Bacaner N, Stauffer B, Boulware DR, Walker PF, Keystone JS, 2004. Travel medicine considerations for North American immigrants visiting friends and relatives. JAMA 291: 2856-2864.

10. LaRocque RC, Rao SR, Tsibris A, Lawton T, Barry MA, Marano N, Brunette G, Yanni E, Ryan ET, 2010. Pre-travel health adviceseeking behavior among US international travelers departing from Boston Logan International Airport. J Travel Med 17: 387-391.

11. Hagmann S, Benavides V, Neugebauer R, Purswani M, 2009. Travel health care for immigrant children visiting friends and relatives abroad: retrospective analysis of a hospital-based travel health service in a US urban underserved area. $J$ Travel Med 16: 407-412.

12. Hagmann S, Reddy N, Neugebauer R, Purswani M, Leder K, 2010. Identifying future VFR travelers among immigrant families in the Bronx, New York. J Travel Med 17: 193-196.

13. Heywood AE, Zwar N, 2018. Improving access and provision of pre-travel healthcare for travellers visiting friends and relatives: a review of the evidence. J Travel Med 25: 1-8.

14. Backer $\mathrm{H}$, Mackell $\mathrm{S}, 2001$. Potential cost-savings and quality improvement in travel advice for children and families from a centralized travel medicine clinic in a large group-model health maintenance organization. J Travel Med 8: 247-253. 